\title{
The Calculation of the Tensely Strained State of the System «Diamond Bit-Bottom»
}

\author{
Aigerim E. Assan ${ }^{1}$, Yury E. Budyukov ${ }^{2}$, Vladislav P. Onishinn ${ }^{3}$, Vitaly V. Povetkin ${ }^{1} \&$ Toktasin M. Mendebayev ${ }^{1}$ \\ ${ }^{1}$ The Kazakh national technical university named after K.I.Satpayev, Almaty, Kazakhstan \\ ${ }^{2}$ Open joint-stock company «Tula scientific-research geologic enterprise» (Open JSC «Tula SRGE»), Tula, \\ Russia \\ ${ }^{3}$ Closed joint-stock company «EZTAB Mining Group»(Closed JSC «EZTAB MG»), Sankt-Petersburg, Russia \\ Correspondence: Aigerim E. Assan, The Kazakh national technical university named after K.I.Satpayev, Almaty, \\ Kazakhstan. E-mail: aigera_ask@mail.ru
}

Received: April 15, 2015

doi:10.5539/mas.v9n11p203
Accepted: May 11, $2015 \quad$ Online Published: September 30, 2015

URL: http://dx.doi.org/10.5539/mas.v9n11p203

\begin{abstract}
The design procedure of the tensely strained state(TSS) of the system «diamond bit-bottom » is developed, basic analytical dependences, characterizing TSS of the system of the calculation the basic parameters-tension, strains under the influence of an external loading on the diamond bit with finite-element method application (FEM) are chosen in the offered article. The questions of the creation in plane posing with usage of share the block schema of the program, realizing finite-element method. For components of the process of interaction with a diamond bit rock used the method of mathematical modeling of components of the process (partial solutions) output data which will be the main characteristics of the process, allow to formulate requirements for the development of the diamond rock cutting tool.

And also addressed the issues of creating a removable drill bit used for boring exploration wells are considered in the article. The authors propose a technological solution for the modernization and improvement of removable drill bit, taking into account the various stages of deterioration of diamond bits.

The possibility of improving the diamond destructive tool, including a special on the scientific, engineering and technological level are far from exhausted. Diamond drilling in difficult geological conditions accompanied not enough efficient use of energy supplied to the face, the working face of overheating matrices crowns, abnormal wear of the rock cutting tool, and is not always suitable geological work quality. In this context, the relevance of the set objectives in this paper is quite obvious.
\end{abstract}

Keywords: Bottom, hole, drilling, diamond bit, diamond drilling, tension, strain, modular of elasticity, finite-element method

\section{Introduction}

The high technical and economic efficiency of the implementation of a set of special diamond rock-breaking tool confirmed practical application at the largest deposits of ores and coal in Russia and CIS.

Well drilling is the basic way of minerals prospecting. Thus the most effective now and the foreseeable future in the way of holes construction in hard rocks is diamond drilling with application of the diamond rock-destruction tool intended forcore drilling by progressive means in difficult mining and geological conditions: column shells with retrievable core receivers (CSRCR), complexes for drilling with core hydraulic transport (CHT), single column shells with application of compressed air and froths, jet shells, and also for noncore and a guided drilling without wedge shells.

For the purpose of power consumption processreducing by drilling andincreasing working capacity of the special diamond rock-destruction tool it is necessary to value performance of combined action of several diamond grains. Therefore the great value gets substantiation of the relative positioning of diamond cutters and spacing interval between them. With this end in view there were held analytical and experimental researches of process of forcing of the indenter to formation. 


\section{The Research Technique}

Let's consider as a whole the system stressed state «a diamond bit bottom». Taking into account that this system is characterized by residual elastic properties(FEM) and so consists of the materials with very different on physical-mechanical properties, for calculation its stressed state the finite-element method (FEM)matches more which one is a modern method of engineering and mathematical problems solution with application of computers .

In a three-dimensional Cartesian reference system $x, y$ and $z$ stressed state is characterized by stresses $\sigma_{x}, \sigma_{y}$, $\sigma_{z}, \tau_{x y}, \tau_{y z}, \tau_{z x}$ and deformed - strains $\varepsilon_{x}, \varepsilon_{y}, \varepsilon_{z}, \gamma_{x y}, \gamma_{y z}, \gamma_{z x}$ - - Nature of the dependences linking tension and strain in a dot of environment determines its behavior under loading [6].

According to closed loop system of the equations characterizingtensely strained state in the body, includes:

The differential equations of equilibrium

$$
\left.\begin{array}{c}
\frac{\partial \sigma_{x}}{\partial x}+\frac{\partial \tau_{x y}}{\partial z}+\frac{\partial \tau_{x z}}{\partial z}=0 \\
\frac{\partial \tau_{x}}{\partial x}+\frac{\partial \sigma_{y}}{\partial z}+\frac{\partial \tau_{y z}}{\partial z}=0 \\
\frac{\partial \tau_{x z}}{\partial x}+\frac{\partial \tau_{y z}}{\partial z}+\frac{\partial \sigma_{z}}{\partial z}=0
\end{array}\right\}
$$

Equations of connection of strains and relocation (Cauchy relations)

$$
\left.\begin{array}{l}
\varepsilon_{x}=\frac{\partial V_{x}}{\partial x} ; \\
\varepsilon_{y}=\frac{\partial V_{y}}{\partial y} ; \\
\varepsilon_{z}=\frac{\partial V_{z}}{\partial z} ; \\
\gamma_{x y}=\frac{\partial V_{x}}{\partial y}+\frac{\partial V_{y}}{\partial x} ; \\
\gamma_{y z}=\frac{\partial V_{y}}{\partial z}+\frac{\partial V_{z}}{\partial y} ; \\
\gamma_{x y}=\frac{\partial V_{z}}{\partial x}+\frac{\partial V_{x}}{\partial z} ;
\end{array}\right\}
$$

Physical proportions of connection of tensely strained state (Hooke's law)

$$
\left.\begin{array}{l}
\varepsilon_{x}=\frac{1}{E}\left[\sigma_{x}-\mu\left(\sigma_{y}+\sigma_{z}\right)\right] ; \\
\varepsilon_{y}=\frac{1}{E}\left[\sigma_{y x}-\mu\left(\sigma_{z}+\sigma_{x}\right)\right] ; \\
\varepsilon_{z}=\frac{1}{E}\left[\sigma_{z}-\mu\left(\sigma_{x}+\sigma_{y}\right)\right] ; \\
\gamma_{x y}=\frac{2(1+\mu)}{E} \tau_{x y} ; \\
\gamma_{y z}=\frac{2(1+\mu)}{E} \tau_{y z} ; \\
\gamma_{x z}=\frac{2(1+\mu)}{E} \tau_{x z} ;
\end{array}\right\} ;
$$

Where. $E$ Modular of elasticity of the first rate (elastic modulus); $\mu$ A Poisson's ratio.

By the solution of static problems the form and the sizes of the body, its position in interspace,constant resiliency are set. The kinematic boundary conditions (replacement of each dot of the surface of the body), or static 
boundary conditions (surface stresses in each dot of the surface of the body), or the blended boundary conditions (on the part of the surface of the body relocation are set, and on the other part of the surface - stresses) are set [7].

In view of that direct system integration of the equations (1-3) represents principal difficulties, we use equivalent variation problem $[8,9]$.

According to the theorem of potential energy from all relocationmeeting kinematic boundary conditions, permanent (extreme) value of potential energy dispatches those relocations which meet the equilibrium equations.

Complete potential energy of the elastic body we introduce in the following way:

$$
\Pi=0,5 \int_{V} \sigma_{i j} \varepsilon_{i j} d V-\int_{S_{f}} F_{i} V_{i},
$$

Where $\sigma_{i j}$-- constituents of the tensor stresses, $\varepsilon_{i j}$ - constituents of the tensor strains, $V$ - volume of the body, $s_{f}$ - part of the surface body to which is enclosed the external loading $F_{i}$.

A number of processes proceed in conditions when strain in one of coordinate directions is insignificant also it is possible to take over approximately equal to zero point. In this case the plane strained state takes place. In case $\varepsilon_{z}=0$ tensely strained state is characterized by following magnitudes: $\sigma_{x}, \sigma_{y}, \sigma_{z}, \tau_{x y}, \varepsilon_{x}, \varepsilon_{y}, \gamma_{x y}$.

Further we use matrix notation of expressions for a variety of causes. In case of plane deformation corresponding column vector havefollowing appearances (the constituents which are not bringing the contribution to potential energy of system, are pulled down)

$$
\begin{array}{r}
\{\sigma\}^{T}=\left[\sigma_{x} \sigma_{y} \tau_{x y}\right] \\
\{\varepsilon\}^{T}=\left[\varepsilon_{x} \varepsilon_{y} \gamma_{x y}\right]
\end{array}
$$

The Hooke's law in matrix notation will assume followingstructure

$$
\{\sigma\}=[D]\{\varepsilon\},
$$

Where matrix $[D]$ - is a square symmetric matrix dimension $3 \times 3$ :

$$
[D]=\frac{E}{(1-2 \mu)(1+\mu)}\left[\begin{array}{ccc}
1-\mu & \mu & 0 \\
& 1-\mu & 0 \\
\text { cuм } & & \frac{1-2 \mu}{2}
\end{array}\right]
$$

The numerical analysis in plane posing was held with the application of finite element (FE) in the form of triangular simplexes-elements. Such elements were widely used in kind of some advantages: simplicity of approximating functions, possibility of exception of routines of numerical integration by reception certain element equations, flexibility at submission of geometrical fields of the difficult form, satisfaction to requirements of inter element integrity, stability, convergence and completeness $[8,9,10]$.

The reception of matrixes $[N],[B]$ and columnvector $\{F\}$, which used in admitting finite element equations.

The relocation in coordinatedirections xuyandwithin a finite element are determined by constituents $V_{x}$ and $V_{y}$,which is necessary to express through central constituents of relocations. The relocationseach knot has two constituents and thus, in the finite element of relocation is determined uniquely by means of six magnitudes.

If fitting $V_{x}$ и $V_{y}$ via two linear polynomials

$$
\left.\begin{array}{l}
V_{x}=\alpha_{1}+\alpha_{2} x+\alpha_{3} y \\
V_{y}=\alpha_{4}+\alpha_{5} x+\alpha_{6} y
\end{array}\right\}
$$

that after some transforming we receive in matrix kind 


$$
\left\{\begin{array}{l}
V_{x} \\
V_{y}
\end{array}\right\}=\left[\begin{array}{l}
N_{i} 0 N_{j} 0 N_{k} 0 \\
0 N_{i} 0 N_{j} 0 N_{k}
\end{array}\right]\left\{\begin{array}{l}
v_{x_{i}} \\
v_{y_{i}} \\
v_{x_{j}} \\
v_{y_{j}} \\
v_{x_{k}} \\
v_{y_{k}}
\end{array}\right\}
$$

Or in compact record

$$
\{V\}=[N]\{v\}
$$

Where $v_{x_{i}}, v_{y_{i}}, \ldots, v_{y_{k}}$ - central constituents of relocations in the direction of axisxиy; $N_{i}, N_{j}, N_{k}$ functions of the element forms, calculating on the expressions

$$
\begin{gathered}
N_{i}=\frac{1}{2 S_{i j k}}\left(a_{i}+b_{i} x+c_{i} y\right) \\
a_{i}=X_{j} Y_{k}-X_{k} Y_{j} ; \\
b_{i}=Y_{j}-Y_{k} ; \\
c_{i}=X_{k}-X_{j} ;
\end{gathered}
$$

(for $N_{i}$ and $N_{k}$ expressions are determined similarly taking into account cyclic shuffle of index markin chain $i$, $j, k)$;); - the delta space $i j k$, determining under the formula

$$
S_{i j k}=\left(X_{j} Y_{k}+X_{k} Y_{i}+X_{i} Y_{j}-X_{j} Y_{i}-X_{i} Y_{k}-X_{k} Y_{j}\right) / 2
$$

The bonding relationship between strains and relocations taking into account (2.2), (2.10) and (2.11), can be introduced in followingway

$$
\left\{\begin{array}{l}
\varepsilon_{x} \\
\varepsilon_{y} \\
\gamma_{x y}
\end{array}\right\}=\frac{1}{2 S_{i j k}}\left[\begin{array}{cccccc}
b_{i} & 0 & b_{j} & 0 & b_{k} & 0 \\
0 & c_{i} & 0 & c_{j} & 0 & c_{k} \\
c_{i} & b_{i} & c_{i} & b_{j} & c_{k} & b_{k}
\end{array}\right]\{v\}
$$

As functions of relocation inside FE linear, strains will be constants within the element.

$$
\{\varepsilon\}=[B]\{v\}
$$

The stresses arising at operating of exterior forces are routinely considered as the concentrated forces in knots FE, equivalent by the distributed stresses, and recorded by means of expression

$$
\{F\}=\int_{S}\left[N_{1}\right]^{T}\{f\} d S
$$

The matrix $\left[N_{1}\right]$ in difference from $[N]$ contains zero values for form functions in those knots which do not lie on the element side where stress is considered. For example, if to the side of the element $i j$ is distributed load with constituents $p_{x}$ и $p_{y}$ on axis $x$ и $y$, that expression for $\{F\}$ poses 


$$
\{F\}=\int_{S_{i j}}\left[\begin{array}{cc}
N_{i} & 0 \\
0 & N_{i} \\
N_{i} & 0 \\
0 & N_{i} \\
0 & 0 \\
0 & 0
\end{array}\right]^{T}\left\{\begin{array}{l}
p_{x} \\
p_{y}
\end{array}\right\} d S=\frac{L_{i j}}{2}\left\{\begin{array}{l}
p_{x} \\
p_{y} \\
p_{x} \\
p_{y} \\
0 \\
0
\end{array}\right\}
$$

Where $L_{i j}$ - length of the element sides between knots $i$ and $\mathbf{j}$.

From received for matrix $[B]$ expressions follows that it does not depend on coordinate position that allows executing simply integration in certainly element equations. For example, calculus of volume integral is executed as follows

$$
\int_{V_{i j k}}[B]^{T}[D][B] d V=\int_{S_{i j k}}[B]^{T}[D][B] t d x d y=[B]^{T}[D][B] t S_{i j k}
$$

Where $t$ is thickness of the finite element. Typically in tasks of plane deformation is taken over $\mathrm{t}=1$.

Using (4), (7), (14) and (15) we record expression for complete potential energy with reference to a finite element

$$
\Pi^{e}=\int_{V^{e}}\left\{v^{e}\right\}^{T}\left[B^{e}\right]^{T}\left[D^{e}\right]\left[B^{e}\right]\left\{v^{e}\right\} d V-\int_{S_{f}^{e}}\left[v^{e}\right]^{T}\left[N_{1}^{e}\right]^{T}\left\{f^{e}\right\} d S
$$

The minimization of complete potential energy of all system consisting from E of elements is executed according to the following routine

$$
\frac{\partial}{\partial\left\{v_{k}\right\}} \sum_{e=1}^{E} \Pi^{e}=\sum_{e=1}^{E} \frac{\partial \Pi^{e}}{\partial\left\{v^{e}\right\}}=0
$$

where is a number of global freedom degrees (number of replacement constituents) for all system as a whole. Including for a separate finite element it leads to the following equation

$$
\Pi^{e}=\int_{V^{e}}\left[B^{e}\right]^{T}\left[D^{e}\right]\left[B^{e}\right] d V\left\{v^{e}\right\}-\int_{S_{f}^{e}}\left[v^{e}\right]^{T}\left[N_{1}^{e}\right]^{T}\left\{f^{e}\right\} d S=0
$$

Last expression represents system of the linear algebraic equations (the index of attachment to a separate element is pulled down for record simplification):

$$
[K]\{v\}-\{F\}=0
$$

where $[K]$ is matrix of rigidity of the finite element:

$$
[K]=\int_{V^{e}}\left[B^{e}\right]^{T}\left[D^{e}\right]\left[B^{e}\right] d V
$$

$\{F\}$ is column vector allowing for exterior applied load (for those elements on which on sides is presented given constituent)

$$
\{F\}=\int_{S_{f}}\left[N_{1}\right]^{T}\{f\} d S
$$

As a result of applied above routine finite elementof the digitization complete potential energy is represented, thus, as function of the confined number of parameters is functions of replacement in knots of elements.

It is necessary to notice that the resulted routine of constructing of permissive system of the algebraic equations with usageof mathematical character of summation in FEM is not summation in the taken meaning, and 
designates so-called assembling operation at which by performance each local matrix or localcolumn vector (referred into separate FE) take place in global matrix or globalcolumn vector (referred to all field) according to numbers of knots of considered FE.

As a result of a set of equations solution (20) we receive a vector of replacement in knots of finite elements $\{v\}$. Then by means of proportion (14) we determine constituents of strains and, at last, using expressions (7), we calculate stresses in finite elements.

The developed finite element equations can be applied to the analysis of the is tensely strained state of the system including some materials with various mechanical performances that allows to execute complex analysis of the tensely strained state of the system «a diamond bit bottom».

\section{Experimental Research}

The calculation of the tensely strained state of the system «a diamond bit bottom».

Let's execute calculation tensely strained state of the system «a diamond bit bottom» with usage of the design schema, it is introduced

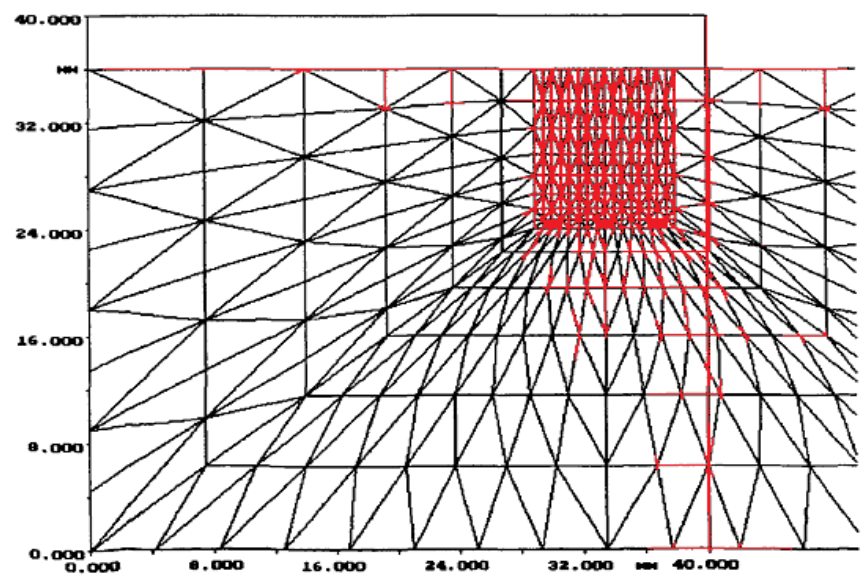

Figure 1. The design schema of the system «a diamond bit bottom»

Here environments with excellent from each other mechanical performances are introduced: drill bit, a diamond pane, the formation, the broken deformation. All system was modeled with usage of 640 finite elements (FE).

The problem was solved in plane posing with usage of share the block - schema of the program, realizing finite-element method (Figures 2-3).

The boundary conditions were set as follows: on the bottom and side boundaries - $v_{x}=0, v_{y}=0$. The effort is made to the upper boundary of the drill bit $P=6,2 \mathrm{DBa}$, operating along axis at that taking into account the floor space of the end part of the drill bit builds up $1200 \mathrm{~kg}$.

In Figure 4 intensity distribution of magnitudestresses on the finite elements, received as a result of elastic deformation of all considered system is demonstrated.

$$
\sigma_{u}=\frac{1}{2} \sqrt{\left(\sigma_{x}-\sigma_{y}\right)^{2}+\left(\sigma_{y}-\sigma_{z}\right)^{2}+\left(\sigma_{z}-\sigma_{x}\right)^{2}+6\left(\tau_{x y}^{2}+\tau_{y z}^{2}+\tau_{z x}^{2}\right)}
$$

On the right of the Figure the scale of unit value for solution in the plane posing, corresponding to various colour displays in the Figure under condition of splitting of unit values of the investigated magnitude into 10 distances is resulted.

From the data of the Figure 4 it is visible that after the offloading appendix in the set tool part of the greatest stress values reach on the ground adjoining to boundary of exterior affecting, that is on contact of the matrix with the drill bit body. Here intensity magnitudes of shearing stresses reach values in 2,1-3,9 times more than in the fields flanking to side walls and matrix end of the drill bit for which one values of the shearing stresses lie in the distance of 1,6 3,9 DBa. By using of the correspondingcriteria of hardness in the given approach, it is possible to determine during what moment there will come breaking down of formations under the drill bit. From the position of brittle failure of the formation in the conditions of difficult stressed stateare the most 
eligible criterion Balandin, Kulon-Mora and Pisarenko-Lebedev.

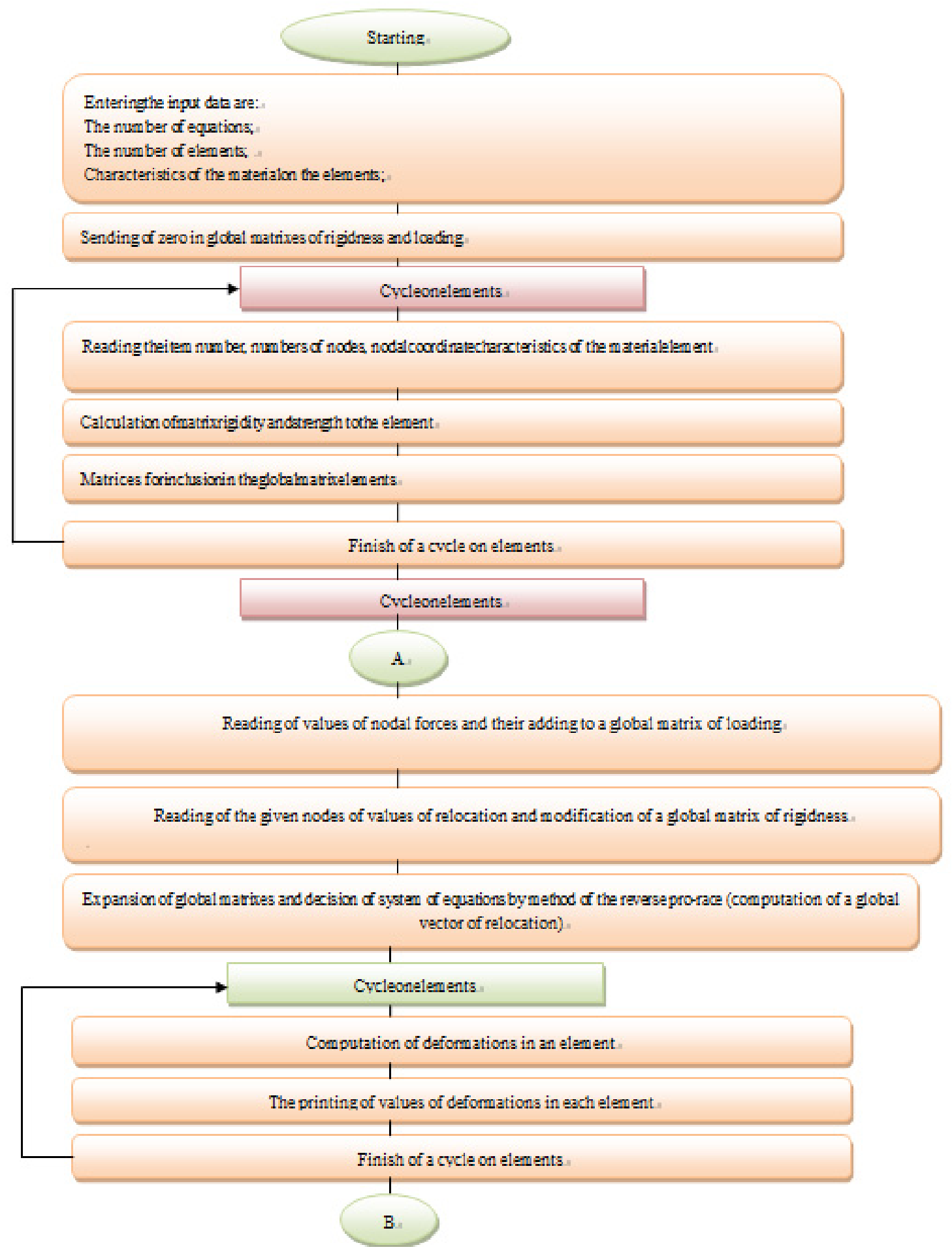

Figure 2. The common block diagramme of the program realizing a finite-element method, cycles A, A-B 


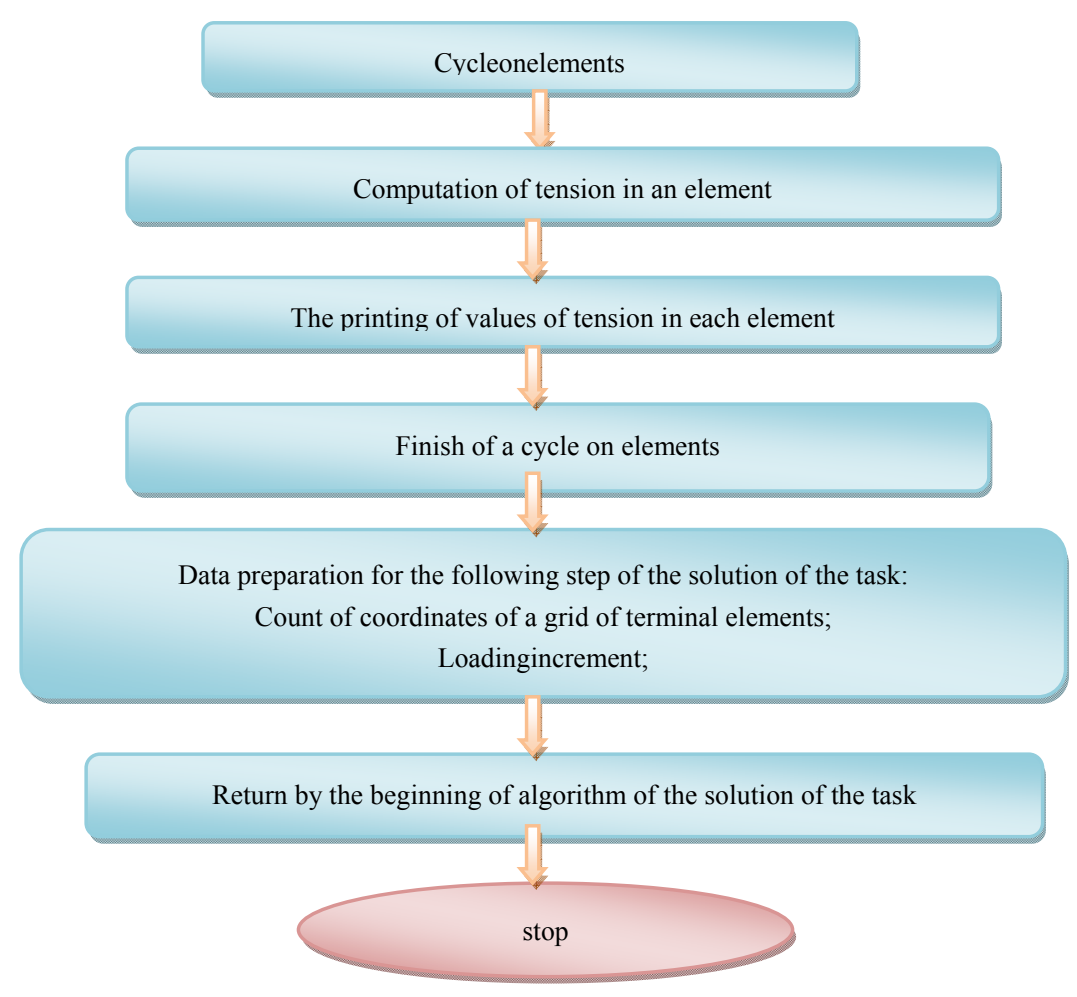

Figure 3. The common block diagramme of the program realizing a finite-element method-cycle B

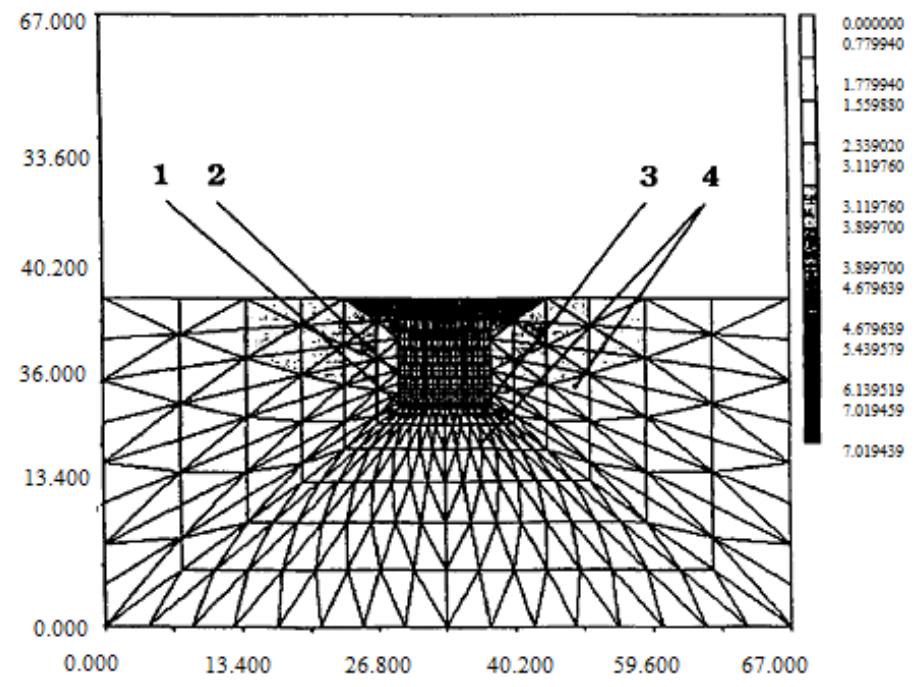

Figure 4. Magnitude distribution $\sigma_{u}$ in thecalculated field

1 - diamonds; 2 - drill bit matrix; 3 - the broken deformation; 4 - monolithic deformation

With application of the developedis investigated distribution method of intensity of the shearing stresses in the deformation (granite) at simultaneous affecting on it with normal offloading of three (Figure 5) indenters of the spherical form close to the form of diamond grains.

As it is evident from Figure 5 with stress offloading magnification inthe deformation increase in the operating zone side indenters more intensively, than in the field of the appendix of central indenter and on some depth merges with each other and come close to the field of central indenter stresses. Controlling distance between 
indenters, it is possible to enhance performance of deformation breaking have served as a substantiation of the schema of diamonds apportion in special single-layer diamond bits and engineering of drilling practices.

a

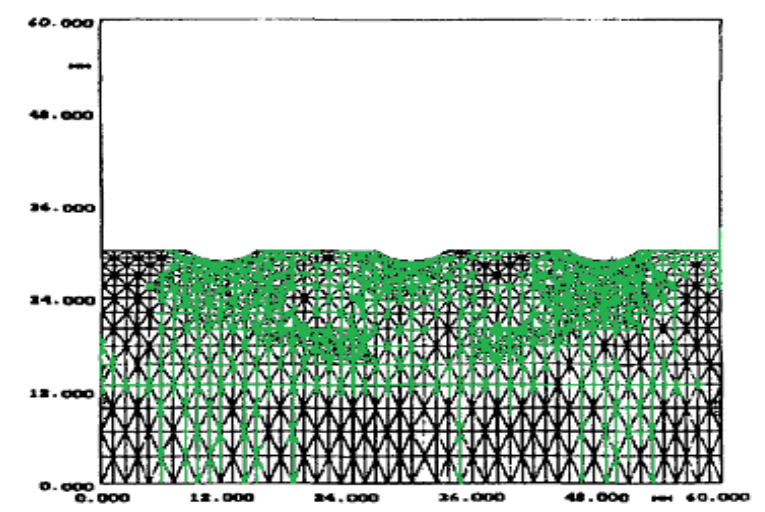

b

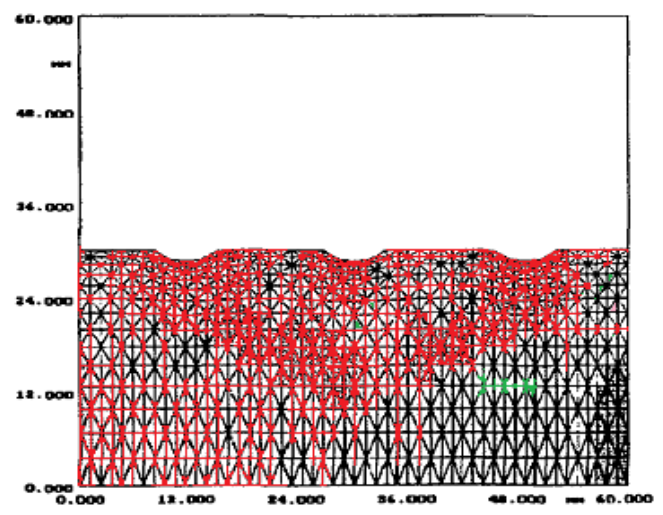

Figure 5. The stress distributionbyloading 3 diamond grains a - loadingconceptual state; $\sigma$ - loading final stage

\section{Conclusions}

On the basis of the held studies of the tensely- strained state of the bottom and diamond tool by boring it is possible to make following conclusions:

1. The analytical dependences describing VAT of the system «diamond drill bit-bottom» including differential equation equilibriums of the deformation connection equation and physical proportions of connections of the tightened and strained state are introduced.

2. The certainly-element method of studies of the tensely - strained state of the system «diamond drill bit-bottom» is developed,allowing to value influence of offloading on the stress distributionnature in the deformation and in elements of deformation breaking drill bit parts.

3. The intensity distribution of shearing stresses in the deformation at simultaneous affecting on it with a normal offloading of three indenters of the spherical form close to the form of diamond grains is investigated.

4. With magnification ofthe stress offloading in the deformation increase in the field of side indenters more intensively than the field of the appendix of central indenter and on some depth merges with each other and come close to the field of central indenter stresses. Controlling distance between indenters, it is possible to enhance performance of deformation breaking.

5. The formation stressed state of the deformation on the condition of Mohr hardness at simultaneous affecting on it with normal and a shearing offloading of two diamond cutters of the spherical form is 
investigated.

\section{References}

Agapov, V. P. (2000). The Finite Element Method in statics, dynamics and spatial stability of thin-walled reinforced constructions. Textbook / M.: DIA, - $152 \mathrm{p} .:$ Ill.

Budyukov, Y. E., Vlasyuk, V. I., \& Seledkin, E. M. (2003). Calculation of tense-deformed state of the "diamond crown-slaughter" within and chapels elastic finite element method. VIEMS deposited. MG02. number 1190-M.:, p.12.

Franca, L. F. P., Mostofi, M., \& Richard, T. (2015). Interface laws for impregnated diamond tools for a given state of wear. International Journal of Rock Mechanics and Mining Sciences.

Ghasemloonia, A., \& Rideout, D. G. (2013). Butt, S.D. Journal of Energy Resources Technology, Transactions of the ASME Vibration analysis of a drillstring in vibration-assisted rotary drilling. Finite element modeling with analytical validation, 135(3), Article number 032902.

Hareland, G. (2012). Calculation of friction coefficient and downhole weight on bit with finite element analysis of drillstring, Volume 4, Pages 2360-2365, 46th US Rock Mechanics / Geomechanics Symposium 2012; Chicago, IL; United States; 24 June 2012 through 27 June 2012; Code 95262

Kalinin, A. G., Vlasyuk, V. I., Oshkordin, O. V., \& Scriabin, R. M. (2004). Drilling Technology. - M.: Publishing "Technology" TUMAGRUPP, $528 \mathrm{p}$.

Makaryants, G. M., \& Prokofiev, A. B. (2013). Fundamentals of finite element method. Textbook-Samara Univ Samaria. Gos. aerokosm. University Press, 80p.: Ill.

Mustapha, K. B., \& Zhong, Z. W. (2013). A new modeling approach for the dynamics of a micro end mill in high-speed micro-cutting, JVC. Journal of Vibration and Control, 19(6), 901-923. Advanced Science Letters Pages, 141-146.

Spirin, V. I., \& Levine, D. M. (2000). creating new lines of diamond porodorazrushayushego tool. Tula, State University. -Tula.: - 149, il ..

Vlasyuk, V. I., Budyukov, Y. E., Gorshkov, L. K., Ossetski, A. I., \& Rjabchikov, S. A. (2002). new technologies in the creation and use of the diamond tool porodorazruschayuschego. M.: CJSC "Geoinformmark, NSU". $140 \mathrm{p}$.

Yu, P. P., Huang, B. W., Tseng, J. G., Tseng, J. K., \& Zheng, J. R. (2012). A study on cutting property in micro drilling process. Geotechnical and Geological Engineering, 30(1), 145-161.

\section{Copyrights}

Copyright for this article is retained by the author(s), with first publication rights granted to the journal.

This is an open-access article distributed under the terms and conditions of the Creative Commons Attribution license (http://creativecommons.org/licenses/by/3.0/). 